\title{
Cholinergic Deafferentation of the Entorhinal Cortex in Rats Impairs Encoding of Novel But Not Familiar Stimuli in a Delayed Nonmatch-to-Sample Task
}

\author{
Jill McGaughy, Randal A. Koene, Howard Eichenbaum, and Michael E. Hasselmo \\ Department of Psychology Center for Memory and Brain, Program in Neuroscience, Boston University, Boston, Massachusetts 02215
}

\begin{abstract}
Acetylcholine may regulate working memory for novel stimuli by activating intrinsic mechanisms for sustained spiking in entorhinal cortical neurons, which have been demonstrated in slice preparations of the entorhinal cortex. Computational modeling demonstrates that loss of the cholinergic activation of intrinsic mechanisms for sustained activity could selectively impair working memory for novel stimuli, whereas working memory for familiar stimuli could be maintained because of previously modified synapses. Blockade of muscarinic cholinergic receptors and selective cholinergic lesions has been shown to impair encoding in delayed matching tasks. However, previous studies have not compared explicitly the role of cholinergic modulation in working memory for novel versus familiar stimuli. Here, we show that lesions of the cholinergic innervation of the entorhinal cortex selectively impair delayed nonmatch to sample performance for novel odors, whereas delayed nonmatch to sample for familiar odors is spared. This indicates an important role for cholinergic innervation of the entorhinal cortex in working memory for novel stimuli.
\end{abstract}

Key words: acetylcholine; episodic buffer; sustained activity; computational modeling; working memory; persistent spiking

\section{Introduction}

Acetylcholine (ACh) may regulate working memory mediated by the entorhinal cortex (EC). In entorhinal cortex slices, acetylcholine causes neurons to respond to a single depolarizing event with sustained spiking activity, resulting from activation of an intrinsic cation current (Klink and Alonso, 1997a; Egorov et al., 2002). Models show how this sustained spiking activity could underlie working memory (Lisman and Idiart, 1995; Jensen and Lisman, 1996; Fransen et al., 2002; Koene et al., 2003), including spiking activity in the EC associated with performance of delayed matching tasks (Suzuki et al., 1997; Young et al., 1997; Fransen et al., 2002). Consistent with this, blockade of muscarinic cholinergic receptors impairs delayed match-to-sample performance (Bartus and Johnson, 1976; Penetar and McDonough, 1983).

The role of acetylcholine in working memory may be particularly important for novel stimuli. The sustained spiking induced by acetylcholine in entorhinal cortex slices occurs despite blockade of synaptic transmission (Klink and Alonso, 1997a; Egorov et al., 2002). Models show how this allows working memory for

Received June 10, 2005; revised Sept. 26, 2005; accepted Sept. 27, 2005.

This work was supported by National Institute of Mental Health Grants 60013, 61492 (M.E.H.), and 60450, National Science Foundation Science of Learning (enter Grant SCL SBE 0354378, and National Institute on Drug Abuse Grant 16454 as part of the Collaborative Research in Computational Neuroscience Program (H.B.E., M.E.H.). We thank Mayuri Jindal for excellent technical assistance in portions of the behavioral training.

Correspondence should be addressed to Michael E. Hasselmo, Department of Psychology Center for Memory and Brain, Program in Neuroscience, Boston University, 2 Cummington Street, Boston, MA 02215. E-mail: hasselmo@bu.edu.

J. McGaughy's present address: Department of Psychology, University of New Hampshire, Conant Hall, 10 Library Way, Durham, NH 03824. E-mail: j.mcgaughy@unh.edu.

DOI:10.1523/JNEUROSCI.2386-05.2005

Copyright $\odot 2005$ Society for Neuroscience ～0270-6474/05/2510273-09\$15.00/0 novel input patterns (Lisman and Idiart, 1995; Jensen and Lisman, 1996; Fransen et al., 2002; Koene et al., 2003) without the dependence on modified synaptic connections used in models of prefrontal cortex (PFC) working memory for familiar stimuli (Lisman et al., 1998; Durstewitz et al., 2000). This effect of acetylcholine could provide a buffer for novel stimuli in the entorhinal cortex that facilitates long-term encoding in the hippocampus (Jensen and Lisman, 1996; Baddeley, 2000; Koene et al., 2003). Consistent with this, blockade of cholinergic receptors impairs encoding of visual stimuli for subsequent recognition performance (Aigner and Mishkin, 1986; Aigner et al., 1991; Sherman et al., 2003), and this impairment occurs selectively with local infusion of scopolamine in the perirhinal (PRh) cortex adjacent to the entorhinal cortex (Tang et al., 1997). Functional magnetic resonance imaging studies show activity in parahippocampal structures during working memory for novel stimuli but not familiar stimuli (Stern et al., 2001), which correlates with subsequent long-term memory for these novel stimuli (Schon et al., 2004), and is reduced by systemic administration of scopolamine (Schon et al., 2005). A previous study showed that selective lesions of cholinergic innervation of the rhinal cortex impairs delayed nonmatching for trial-unique stimuli in monkeys (Turchi et al., 2005), but that study did not compare effects on familiar stimuli and did not target the entorhinal cortex.

Although these studies support the hypothesis that cholinergic modulation in the entorhinal cortex subserves the encoding of novel stimuli, none of the previous studies have assessed selective cholinergic lesions of the entorhinal cortex and compared the effects on memory for familiar and novel items. Here, we show that lesions of the cholinergic innervation of entorhinal cortex in rats selectively impair delayed nonmatch-to-sample (DNMS) 
performance for novel odors, whereas delayed nonmatch-tosample for familiar odors is spared. We also present a computational model that demonstrates how loss of cholinergic modulation could selectively impact working memory for novel but not familiar stimuli. These results support an important role for cholinergic innervation of the entorhinal cortex in working memory for novel stimuli.

\section{Materials and Methods \\ Overview}

This experiment focused on comparing the performance of rats on delayed nonmatch to sample with familiar versus novel odors. Rats were trained with 30 odors defined as "familiar" odors until they reached criterion performance before surgery (Dudchenko et al., 2000). For each trial, rats were presented with a sample in which they found reward underneath sand mixed with a sample odor in a cup. After a minimal delay, they were presented with a test trial with a pair of cups, one containing the sample odor and one containing the nonmatch, and were rewarded for choosing the nonmatching stimulus. Fourteen days after surgery, rats were tested with familiar odors with prolonged delays between the sample stimuli and choice or with lists of multiple samples followed by a sequence of choice trials (Dudchenko et al., 2000). After testing with familiar odors, rats were tested in the same task with 24 novel odors. Details of the methods used for this study are provided below.

\section{Subjects}

The study commenced with 16 male Long-Evans rats (Charles River, Boston, MA) weighing between 250 and $300 \mathrm{~g}$ at the beginning of behavioral training. Rats were moderately food deprived but allowed $18 \mathrm{~g}$ of rat chow per diem and ad libitum water to allow them to maintain body weights at $90 \%$ of age-matched controls. Rats were housed separately and kept on a $12 \mathrm{~h}$ light/dark cycle and tested during the light phase of their cycle.

\section{Apparatus and materials}

All training and testing was conducted on a $3 \times 3$ foot square of black Plexiglas surrounded by a 1.25 -inch-tall border. Odors mixed with sand (as described below) were presented in plastic cups (2.625 in diameter; Nalge Nunc, Rochester, NY). These cups were affixed to the platform with Velcro strips to prevent the subjects from tipping their contents. Cups could be placed in the center and along 24 equally spaced locations on the perimeter.

\section{Shaping}

Initially, rats were trained to dig in cups filled with unscented sand (Dudchenko et al., 2000) placed in the center of the platform and baited with a quarter piece of Froot Loop cereal (Kelloggs, Battle Creek, MI). Subjects required $2-3 \mathrm{~d}$ to reliably retrieve reinforcement and were then trained to dig in scented sand.

\section{Odor training}

When rats were reliably retrieving 10 reinforcements/day they began training in an odor DNMS task. One hundred grams of sand was scented with $1.0 \mathrm{~g}$ of common household spices and products. In the sample phase of the task, a scented cup was placed on the center of the platform. The rat was then placed on the platform and allowed to retrieve reinforcement from this cup. Subsequently, the rat was removed from the platform and returned to its home cage while the platform was cleaned with ethanol and an identical cup with the sample scent was placed at a random location with a different odor placed directly across from it. The rat was then placed directly between the two cups with its head oriented perpendicular to the cups. The rat was reinforced for digging in the cup that was different (nonmatch) from the sample. Responses were defined as the rat displacing sand with his forepaw. Initially, rats were trained (10 trials/d) with a subset of 20 odors from a list of 30 . Trials were arranged so that all 30 odors were exposed twice, once as a sample and once as a choice, in three test sessions with a new sequence started after completion of these three sessions. The odors trained before surgery were defined as "familiar" odors, because rats were exposed to them repeatedly before surgery. Rats were trained until they responded correctly on 8 of 10 trials for two consecutive test sessions.

\section{Surgical procedures}

Rats were randomly assigned to receive the immunotoxin 192 IgGsaporin (Advanced Targeting Systems, San Diego, CA) or its vehicle, Dulbecco's saline infused into the region of the entorhinal cortex, using previously published surgical techniques (McGaughy et al., 1996). Before anesthesia, rats were pretreated with $0.25 \mathrm{mg} / \mathrm{kg} / \mathrm{ml}$ dose of atropine to suppress mucousal secretions and produce a patent airway. Rats were anesthetized using halothane gas in a 30:70 oxygen:nitrous oxide mixture. Infusions of the immunotoxin 192 IgG-saporin (Advanced Targeting Systems, San Diego, CA) or vehicle were made into the entorhinal cortex using a 26 gauge Hamilton syringe with a beveled needle angled $10^{\circ}$ from the midline. Coordinates were as follows, with all anteroposterior (AP) measurements relative to bregma, all dorsoventral (DV) measures relative to skull, and the toothbar at -3.3 : AP, -7.8 , mediolateral (ML), $\pm 3.8, \mathrm{DV},-7.2$; AP, -7.3 , ML, $\pm 4.4, \mathrm{DV},-7.4$; AP, $-6.8, \mathrm{ML}$, $\pm 4.0, \mathrm{DV},-8.0 ; \mathrm{AP},-6.3, \mathrm{ML}, \pm 4.4, \mathrm{DV},-8.0$. Infusion volumes were $1.0 \mu \mathrm{l} /$ hemisphere for all sites but the -7.3 in which $0.5 \mu \mathrm{l} /$ hemisphere was used. The infusion needle was left in place $4 \mathrm{~min}$ before and subsequent to the injections to prevent undesired diffusion. An electronic infusion pump (UltraMicroPump II; World Precision Instruments, Sarasota, FL) was used to control infusions (infusion rate of 125 nanoliters/min). Subjects were given ad libitum food and water for $10 \mathrm{~d}$ before the reinstatement of food deprivation procedures and after an additional $4 \mathrm{~d}$, the reinstatement of behavioral testing.

\section{Postoperative testing}

Familiar odors. Rats were tested on the standard DNMS after surgery until they re-established criterion performance. After this time, rats underwent tests with familiar odors to determine whether cholinergic deafferentation of the entorhinal cortex increased sensitivity to increased mnemonic demands produced by prolonged delays between the sample stimuli and choice and by the introduction of lists of multiple samples followed by a sequence of choice trials. Rats were given six trials at each delay $(15,30,60,180 \mathrm{~min})$ and two tests with each list length $(3,5,10,15$ items). On days when delays were tested, the cup was placed in the center of the testing board before the rat being allowed to explore the sample. Subsequent to the retrieval of the reinforcer from the sample cup, the rat and cup were removed from the platform, and the surface was cleaned with a $70 \%$ ethanol solution. The rat was placed in his home cage for the duration of the delay period $(15,30,60$, or $180 \mathrm{~min})$ and returned to the center of the testing platform with its head oriented perpendicular to the testing cups for the assessment of the choice phase including a replication of the sample odor and a nonmatch familiar odor. The side of the nonmatch and position of the cups was randomly varied over the course of the testing session. As before, the rat was reinforced for choosing the nonmatch. Delay trials were tested on separate days in the following order: 15, 60, 30, and $180 \mathrm{~min}$.

In the case of list-length trials, a rat was given sequential presentations of different numbers of sample cups in the center of the platform. Different list lengths were tested on different days in the following order: 5 , 10,3 , and 15 sample items. Immediately after retrieval of the reinforcer from the final cup, the rat was returned to its home cage while the platform was cleaned. The sides of reinforcement and the positions of the choice cups were counterbalanced so that reward was equally likely on either the right or the left side of the board and could occur at any position on the perimeter of the board. Immediately thereafter, the rat was given sequential choice trials for each of the sample odors with the platform cleaned between each of the choice trials.

Novel odors. After testing with familiar odors, rats were given novel odors that had not been used previously in any aspect of testing. The odors used as novel for half of the subjects served as the familiar odors for the other half of the subjects to preclude the possibility that the constructs of novel and familiar were confounded by the odors used. Rats were tested with novel odors at the shortest delay $(15 \mathrm{~min})$ used previously with the familiar odors to allow direct comparisons between these conditions. There were a total of 24 novel odors so that 12 trials could be 
conducted (six trials/day). Breaking the trials over days provided a within-subjects replication of the effects of novel stimuli. Testing continued with each list of novel stimuli being reordered for each session and lists alternated each day over 8 additional days. A retest of the presurgical familiar odors was performed for one session. The following dependent measures were taken for all testing sessions: accuracy (correct choices/all choices), latency to dig in sample cup, latency to correct choice dig, and latency to incorrect choice dig.

Histology. After the completion of all behavioral testing, rats were given an overdose of sodium pentobartital and were transcardially perfused with saline followed by a $10 \%$ buffered formalin. Brains were allowed to sink in a $30 \%$ sucrose solution and then sliced at $50 \mu \mathrm{m}$. Alternate coronal sections were thionin stained for nissl bodies and acetylcholinesterase as optimized for the elucidation of cortical fibers as described previously (Tago et al., 1986). Briefly, sections were placed in phosphate buffer, $\mathrm{pH}$ 7.4, with $0.1 \% \mathrm{H}_{2} \mathrm{O}_{2}$ before rinsing with $0.1 \mathrm{M}$ maleate buffer (three times for $3 \mathrm{~min}$, ph 6.0). Sections were then incubated in a solution of $5 \mathrm{mg}$ of acetylthiocholine iodide, $0.147 \mathrm{~g}$ of sodium citrate, $0.075 \mathrm{~g}$ of copper sulfate, and $0.0164 \mathrm{~g}$ of potassium ferricyanide in 0.1 maleate buffer for $45 \mathrm{~min}$. After incubation, sections were rinsed in $50 \mathrm{~mm}$ Tris buffer (three times for $3 \mathrm{~min}$, ph 7.6). A second incubation then commenced using $0.05 \mathrm{~g}$ of diaminobenzidine and $0.375 \mathrm{~g}$ of nickel ammonium sulfate in $125 \mathrm{ml}$ of $50 \mathrm{~mm}$ Tris buffer. Twelve drops of $0.1 \%$ $\mathrm{H}_{2} \mathrm{O}_{2}$ were added to each $60 \mathrm{ml}$ bath until cortical layers became visible. Sections were rinsed in $5 \mathrm{~mm}$ Tris buffer (three times for $3 \mathrm{~min}$ ) before mounting with gelatin on gelatin-coated slides. Histological assessment of fiber loss was made using a modified grid counting procedure (McGaughy et al., 1996). Sections were examined on an Olympus BX51 microscope using the $40 \times$ objective. A grid measuring $300 \times 250 \mu \mathrm{m}$ was applied to the image of the brain using Image Pro Plus v. 4.5 software (Media Cybernetics, Silver Springs, MD), and the number of fibers that crossed the perimeter of the grid were counted. Fiber counts were obtained in the perirhinal and entorhinal cortices at bregma $-7.8,-7.3$, -6.8 , and $-6.3 \mathrm{~mm}$.

Statistical analyses. All dependent measures were analyzed in separate ANOVAs. The means for each dependent measure were calculated for each delay session over all six trials. The final ANOVA for delay was a mixed factors with one between-subject factor (LESION; two levels) and one within-subject factor (DELAY; four levels). Means for dependent measures for list-length trials were all calculated for each list length and analyzed in a mixed-factor ANOVA with the between-subjects factor, LESION (two levels), and the within-subject factor of LIST LENGTH (four levels). The effects of the first exposure to novel stimuli were compared with familiar stimuli at the same delay in a mixed-factor ANOVA with the between-subjects factor of LESION (two levels) $\times$ NOVELTY (two levels). The effects of repeated exposure to previously novel stimuli were assessed by comparing the effects of LESION (between-subjects factor; two levels) $\times$ DAY (within-subjects factor; four levels). The extent of the lesion was determined in a series of planned $t$ test with the degrees of freedom corrected using a modified Bonferroni.

Computational modeling. We used a simplified simulation of cortical function to demonstrate our proposed mechanism for the selective sensitivity of novel but not familiar stimuli to lesions of the cholinergic innervation of entorhinal cortex. The main components of our model are two integrate-and-fire neuronal networks that simulate memory function in the PFC and the EC. Sensory input about the stimulus in the task causes spiking in a subset of the model neurons in the PFC, as well as a subset of the model neurons in the EC. During the delay period of a delayed nonmatching sample task, a pattern of spiking neurons that represents a familiar stimulus may be maintained in the PFC by the reactivation of the pattern of spikes through recurrent excitatory synaptic transmission. This depends on previous strengthening of excitatory recurrent connections resulting from spike timing-dependent synaptic plasticity. When stimuli are familiar, the animal may draw on the memory maintained in either region to make its decision in the DNMS task.

In contrast, for novel stimuli, a synaptic representation in the PFC does not exist, and we propose that maintenance then depends exclusively on sustained spiking of neurons in the EC maintained by an intrinsic afterdepolarization (ADP) current, which allows persistent spiking
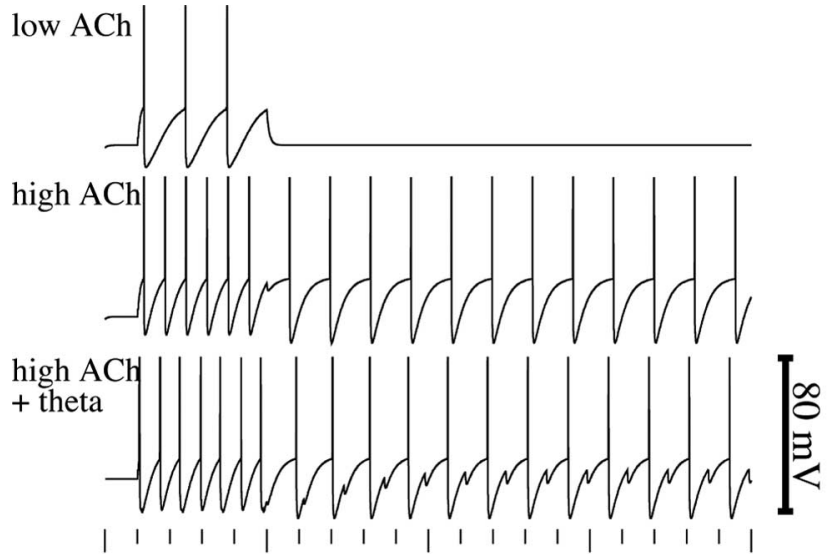

0

1000

$2000 \mathrm{~ms}$

Figure 1. Computational model of the cholinergic activation of sustained spiking activity in entorhinal cortex layer II pyramidal cells resulting from an intrinsic afterdepolarization. Low ACh, In control conditions, entorhinal cortex neurons respond to a short current injection with spiking activity, which ceases when current injection terminates. No persistent spiking occurs after input. High ACh, With activation of muscarinic cholinergic receptors, the spiking activity during current injection causes activation of an intrinsic ADP. After each spike, a brief afterhyperpolarization is followed by an afterdepolarization, which drives membrane potential back up to the spiking threshold so that the pyramidal cell regenerates spikes at regular intervals. This sustained spiking results from intrinsic currents and does not depend on recurrent excitatory synaptic connections. High ACh plus theta, Addition of theta rhythmic depolarization regulates the timing of the repetitive spiking behavior.

without synaptic input. Figure 1 shows a simulation of an intrinsic afterdepolarization current causing sustained spiking, as shown experimentally in cells of the entorhinal cortex during application of a cholinergic agonist (Klink and Alonso, 1997a,b; Egorov et al., 2002). This afterdepolarization results from cholinergic activation of a calcium-sensitive nonspecific cation current, which allows single neurons to regeneratively maintain persistent spiking (Klink and Alonso, 1997a,b; Egorov et al., 2002). Each spike causes calcium influx (through voltage-sensitive calcium channels), which causes additional depolarization which causes another spike. Thus, the intrinsic afterdepolarization in the EC provides an alternate mechanism for sustained spiking, which, in contrast to the PFC, does not depend on modified excitatory recurrent synapses. To make its decision in the DNMS task when stimuli are familiar, the animal may draw on the memory maintained in either region, but for stimuli that are novel, the animal depends on EC activity to perform the task.

We implemented models of PFC and EC neuronal networks and their functions in the CATACOMB2 (Hasselmo et al., 2002a; Cannon et al., 2003; Koene et al., 2003) simulation environment. The simulation used integrate-and-fire neurons for PFC and EC regions and spike timing dependent plasticity (STDP) at synapses in the PFC. Input to the networks was provided in the form of spike events that target specific groups of neurons in both regions for each perceived stimulus. One such pattern of simultaneous spikes initiates each trial of a simulation run. Sustained activity was terminated at the end of each trial in the simulation.

Entorhinal cortex model. In the entorhinal cortex, the sustained spiking is attributable to an ADP of membrane potential that is caused by calcium-sensitive cation currents that are induced by muscarinic cholinergic receptor activation (Klink and Alonso, 1997a,b). This model of sustained spiking for working memory was first proposed by Lisman (Lisman and Idiart, 1995; Jensen and Lisman, 1996) and has been simulated extensively (Fransén et al., 2002; Hasselmo et al., 2002a; Koene et al., 2003). The model used here has similar features to previous simulations (Hasselmo et al., 2002a; Koene et al., 2003). The ADP activated by a spike in the presence of cholinergic modulation is modeled as a dual exponential response with slow rise and fall time constants of $125 \mathrm{~ms}$. As shown in Figure 1, this causes persistent spiking in the model dependent on the presence of acetylcholine.

Our model of the EC contains 64 integrate-and-fire neurons with 
membrane time constants of $9 \mathrm{~ms}$, with resting and reset potentials of $-60 \mathrm{mV}$ and with the ADP. Each individual input pattern activated eight neurons. If sensory input or the ADP causes the membrane potential to cross the threshold of $-50 \mathrm{mV}$, the neuron produces a $1 \mathrm{~ms}$ action potential followed by a $2 \mathrm{~ms}$ refractory period. The spike also initiated a strong afterhyperpolarizing membrane response with a $30 \mathrm{~ms}$ exponential decay time constant and reversal potential of $-90 \mathrm{mV}$. In addition, the spike initiated the ADP mentioned above, with slow rise and fall time constants of $125 \mathrm{~ms}$ and reversal potential of $-45 \mathrm{mV}$. Thus, as the afterhyperpolarization decreases, the ADP depolarizes the membrane toward $-45 \mathrm{mV}$ until it crosses the $-50 \mathrm{mV}$ threshold and causes another spike.

Previous data show that theta rhythm oscillations modulate activity in the entorhinal cortex (Alonso and Garcia-Austt, 1987). This was modeled with $8 \mathrm{~Hz}$ rhythmic input of inhibitory currents with a $-90 \mathrm{mV}$ reversal potential, conductance of $10 \mathrm{nS}$, and dual exponential time course with a rise time of $0.1 \mathrm{~ms}$ and a fall time of $20 \mathrm{~ms}$. The rhythmic modulation maintains the order of the patterns of spiking activity sustained in the EC, and with the aid of recurrent lateral inhibition, it synchronizes the spikes within a pattern and maintains a separation of consecutive patterns.

Recurrent inhibition separates the reactivation of sequential items in such a buffer and is provided by an interneuron representing the population of interneurons that spike in response to pyramidal cell activity. Interneuron resting, reset, and threshold potentials were the same as the excitatory neurons. Previous simulations have shown that the theta rhythmic spiking also synchronizes activity between the entorhinal cortex and hippocampus, in which synaptic modification can form episodic representations for later retrieval (Hasselmo et al., 2002a,b; Koene et al., 2003). Thus, the short period of sustained spiking for novel stimuli simulated here can provide a buffer for encoding episodic representations for longer-term recognition after delays of $15 \mathrm{~min}$ or more.

Prefrontal cortex model. Our model of PFC contains 64 excitatory principal neurons. These integrate-and-fire neurons have membrane time constants of $10 \mathrm{~ms}$ and a reset potential of $-60 \mathrm{mV}$. Crossing the threshold of $-50 \mathrm{mV}$ produces a $1 \mathrm{~ms}$ action potential followed by a $5 \mathrm{~ms}$ refractory period and a strong afterhyperpolarizing membrane response with a reversal potential of $-90 \mathrm{mV}$ that has an exponential decay time constant of $10 \mathrm{~ms}$. A separate pattern of eight PFC neurons spikes for each distinct stimulus input. Recurrent synaptic fibers connect each of the neurons with all other neurons in the PFC. The total synaptic and axonal delay of recurrent transmission is $25 \mathrm{~ms}$. For novel stimuli, the initial strength of the recurrent connections is small so that a pattern of eight spikes causes subthreshold recurrent activation. For stimuli assumed to be familiar at the onset of the simulation, the total synaptic conductance on a recurrent fiber between two neurons in the representative pattern was set to a larger value $(1 \mathrm{nS})$. The membrane response to synaptic input is modeled by a dual exponential function with a $2 \mathrm{~ms}$ rise time and a 4 ms fall time.

With repeated input, our model of PFC can encode novel patterns of activity so that those patterns effectively become familiar patterns. This learning involved STDP at recurrent fiber synapses. The time course of STDP required that presynaptic spikes precede postsynaptic spiking by $<40 \mathrm{~ms}$ as shown in experimental data on Hebbian long-term potentiation (Levy and Steward, 1983; Markram et al., 1997). The STDP learning rate for such presynaptic and postsynaptic spike events was set to 0.3 . We assumed that the stimulus input to the PFC involves a brief burst of spikes so that the presynaptic and postsynaptic timing requirements are met. Multiple trials with the same novel stimulus simulate multiple days of experimental training and testing (see Fig. 6, trials 5-8), which cause strengthening of synapses and allow synapses to cause persistent spiking in PFC on later days.

\section{Results}

\section{Effect of cholinergic lesions on DNMS performance}

Cholinergic lesions of the EC impaired performance of an odor delayed nonmatch-to-sample task for novel but not for familiar odor stimuli. As shown in Figure 2A, lesions of the cholinergic afferents of EC (AChX-EC) impaired delayed nonmatch-to-
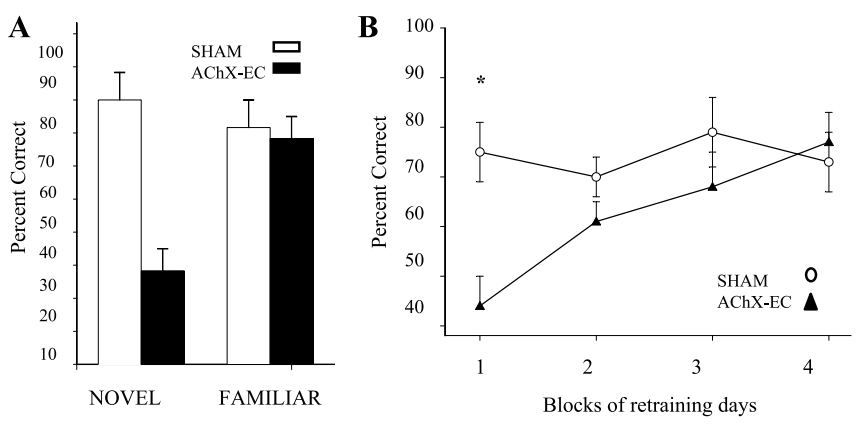

Figure 2. Cholinergic EC lesions impair recognition memory for novel but not familiar odors. $A$, Cholinergic EC-lesioned rats (AChX-EC, filled bars) were severely impaired relative to SHAMs (open bars) in the DNMS at 15 min delay during the first exposure to novel odors (left) but not familiar odors (right). Error bars represent SEM. B, The impairment in processing novel information persisted during the second exposure of the test stimuli, as indicated by the asterisks (EC lesions; filled triangles), but dissipated by the third exposure to the previously novel odors. This suggests that additional repetitions of the novel odors allow formation of representations that do not depend on cholinergic modulation in entorhinal cortex. Later repetitions do not differ from SHAM controls (open circles).
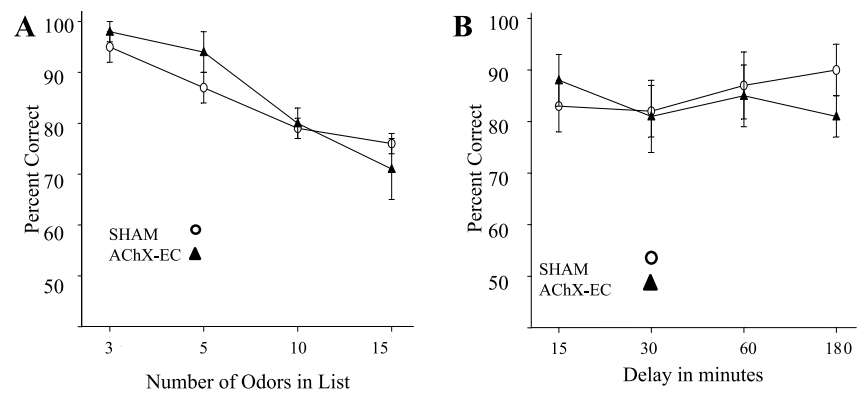

Figure 3. $A$, EC-lesioned rats (filled triangles) were not more sensitive than SHAM controls (open circles) to increases in task difficulty for familiar odors. Both groups show nearly identical impairments when the number of samples to be remembered is increased. This supports the hypothesis that it is the nature of the stimuli (e.g., its novelty), not the cognitive demand, of the task that critically requires the function of the cholinergic afferents to this area. $\boldsymbol{B}, \mathrm{EC}$-lesioned rats (filled triangles) were not more sensitive than SHAM controls (open circles) for increases in delay length for familiar odors. Both groups show nearly identical impairments when a range of delays was tested for familiar odors.

sample performance for novel but not familiar odors at a $15 \mathrm{~min}$ delay $\left(\right.$ AChX-EC $\times$ NOVELTY, $\left.F_{(1,12)}=12.93 ; p=0.004\right)$. As the novel stimuli became familiar over days, the initial accuracy impairment dissipated $\left(\mathrm{DAY}, F_{(3,36)}=3.26, p=0.03\right.$; AChX-EC $\times$ DAY, $\left.F_{(3,36)}=3.33, p=0.03\right)$, as shown in Figure $2 B$. Planned $t$ tests (modified Bonferroni's, 0.013) revealed that lesioned rats were still significantly impaired in remembering novel stimuli after the second day of exposure $\left(t_{(12)}=3.73 ; p=0.003\right)$ but not subsequent days of testing (all, $p>0.09$ ) (Fig. $2 B$ ). There was no effect of novelty or interaction of lesion and novelty on response latency (all, $p>0.05$ ).

Control studies showed that these effects were not caused by selective sensitivity of lesioned rats to task difficulty. As shown in Figure $3 B$, lesions of the EC did not impair accuracy relative to SHAM controls on the test of the memory for familiar stimuli even over long delays (AChX-EC, $F_{(1,12)}=0.15, p=0.70$; AChX$\left.\mathrm{EC} \times \operatorname{DELAY}, F_{(3,36)}=0.77, p=0.52\right)$. As shown in Figure $3 A$, the accuracy of all rats was decreased by a greater number of samples (up to 15 items; $F_{(3,36)}=17.00 ; p<0.001$ ), but lesioned rats were not more sensitive than SHAM controls to these increased mnemonic demands (AChX-EC, $F_{(1,12)}=0.52$; AChX$\mathrm{EC} \times$ LIST LENGTH, $\left.F_{(3,36)}=0.89, p=0.46\right)$. 

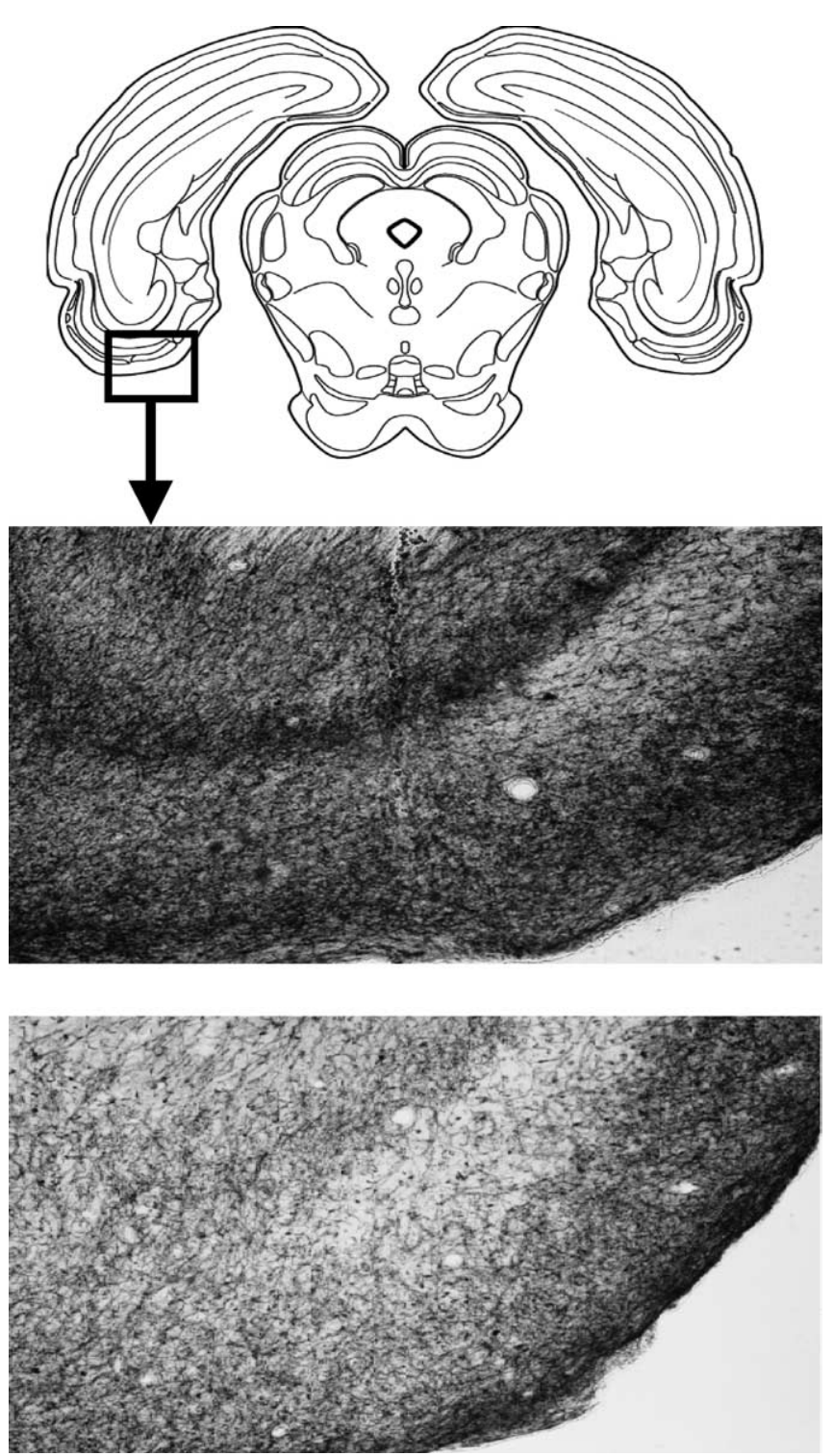

Figure 4. Lesions of the entorhinal cortex. Coronal section of the caudal entorhinal cortex (Swanson, 1999) (bregma, -7.6) that, when damaged, shows a strong positive correlation $(r=0.86)$ with the extent of impairment in working memory for novel information. AChE + fibers from the area highlighted in the inset from SHAM (middle) and AChX-EC (bottom) rats at $10 \times$ magnification. Overall loss as indexed by counts of $A C h E+$ fiber was $63 \%$ across all layers of the cortex.

As shown in Figure 4, the acetylcholinesterase positive $(\mathrm{AChE}+)$ fibers in both the EC and PRh cortex were significantly lower in LESIONED rats relative to SHAM (EC, $t_{(12)}=9.13, p<$ 0.0001; PRh, $\left.t_{(12)}=5.45, p<0.0001\right)$. Means \pm SEMs for fiber counts of LESIONED rats were $113.9 \pm 8.1$ in EC and 151.6 \pm 13.6 in PRh. In contrast, fiber counts in SHAM rats were $303.7 \pm$ 15.6 in the EC and $266 \pm 15.9$ in the PRh cortex. As shown in Figure 5, the extent of damage in the caudal portions of the medial EC was strongly correlated with an impaired performance on DNMS with the first exposure to novel stimuli $(r=0.86 ; p=$ $0.001)$. A Spearman's correlation on data from lesioned animals alone was also significant $(r=0.78 ; p=0.04)$. No correlation was found for performance and damage in the adjacent PRh $(r=$ $0.05 ; p=0.900)$.

In summary, cholinergic deafferentation of the EC selectively impaired rats' ability to encode novel stimuli. This impairment

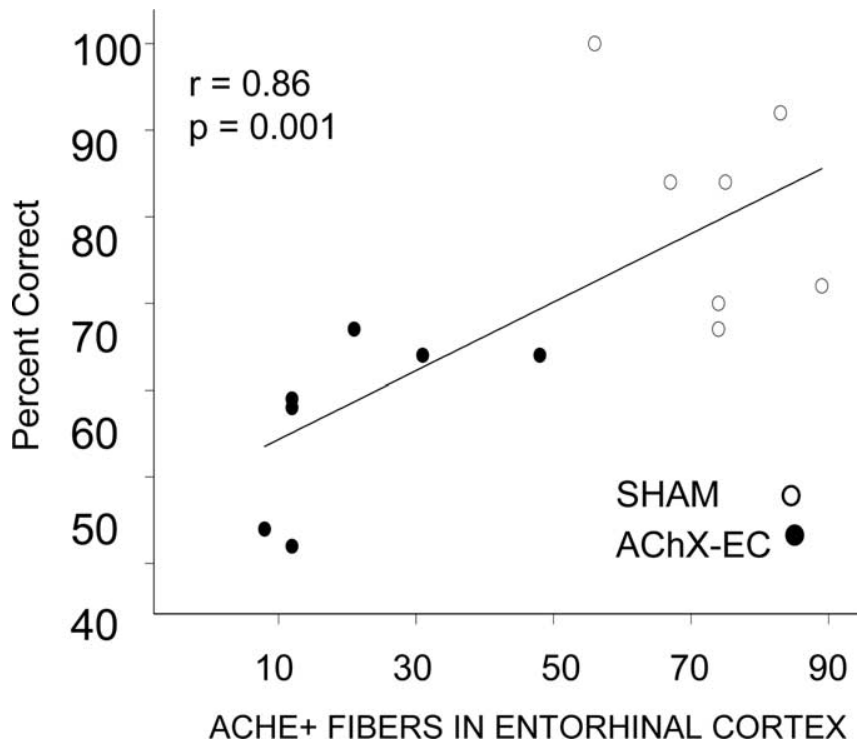

Figure 5. The number of $\mathrm{AChE}+$ fibers in the caudal entorhinal cortex was strongly correlated with accuracy on the DNMS with novel odors $(r=0.86 ; p=0.001$; AChX-EC, filled circles; SHAM, open circles). As noted in Results, this correlation was unique to the entorhinal cortex but not the perirhinal cortex.

was attenuated by repeatedly exposing subjects to these stimuli (i.e., familiarization). This effect does not reflect an increased sensitivity of AChX-EC rats to increases in task difficulty, because AChX-EC rats were not impaired on tests of increased cognitive demand performed with familiar stimuli.

\section{Computational modeling}

Computational modeling demonstrates how buffering of novel stimuli could depend on cholinergic activation of intrinsic mechanisms, whereas buffering of familiar stimuli could depend on previously modified excitatory recurrent connections. Specifically, our computational model shows how loss of cholinergic activation of intrinsic depolarizing currents prevents sustained spiking for stimuli maintained in entorhinal cortex, whereas the PFC can maintain sustained spiking for familiar stimuli through strengthened recurrent synapses independent of cholinergic activation. The same entorhinal buffer model was used in previous simulations (Koene et al., 2003) and is itself a modification of the Lisman-Jensen model for short-term memory (Lisman and Idiart, 1995; Jensen and Lisman, 1996).

Figure 6 shows the membrane potential of neurons in the EC (top two response plots) and the PFC (bottom two response plots) during simulated trials of a DNMS odor task. Each neuron for which the simulated membrane potential is shown in Figure 6 is one member of a set of simulated neurons that fire together to maintain memory of a specific odor stimulus. Initial spikes in the membrane potential of the neurons $\mathrm{EC}_{\text {novel }}$ and $\mathrm{PFC}_{\text {novel }}$ represent the perception of a specific novel odor stimulus in this example, and spikes in the membrane potentials of the neurons $\mathrm{EC}_{\text {familiar }}$ and $\mathrm{PFC}_{\text {familiar }}$ represent the perception of a specific familiar odor stimulus. A simulated train of repeated spikes functions as a memory buffer of a perceived odor stimulus. The simulated activity of neurons in the EC and PFC is shown for eight consecutive sample trials, and each trial is described below. Neural activity is silenced between each trial.

\section{Trial 1}

In simulated trial 1 , the perception of a familiar odor stimulus elicits an initial spike at the time marked by the arrow in neurons 
that are part of the populations $\mathrm{EC}_{\text {familiar }}$ and $\mathrm{PFC}_{\text {familiar. }}$. The spiking activity in these populations then persists after the input stimulus has ceased because of different mechanisms in EC versus PFC. In the EC population, the neurons are reactivated regularly by a combination of the afterdepolarization of membrane potential that follows each spike and the rising phase of rhythmic theta modulation of that membrane potential (Lisman and Idiart, 1995; Jensen and Lisman, 1996; Fransen et al., 2002; Koene et al., 2003). The afterdepolarization depends on cholinergic modulation (Klink and Alonso, 1997a; Egorov et al., 2002). This mechanism can be seen in the plot of $\mathrm{EC}_{\text {familiar }}$ membrane potential, in which both of these effects cause depolarization that follows the rapid hyperpolarization after each spike, resulting in renewed spiking at a theta frequency of $8 \mathrm{~Hz}$. Spikes in the EC are maintained this way without a need for synaptic connectivity. In Figure 6, the repetition of spikes at EC neurons is clearly distinguishable from the repetition of spikes at PFC neurons (which are attributable to excitatory recurrent synapses). Our simulation of EC therefore maintains self-sustained spiking activity for both novel and familiar input stimuli, which may be used directly for matching (Lisman and Idiart, 1995; Fransen et al., 2002) or may enable the slower process of synaptic modification that can encode the episode for matching after a delay (Jensen and Lisman, 1996; Koene et al., 2003).

In contrast to the EC, the simulated PFC maintains persistent spiking only at neurons for which previously strengthened recurrent excitatory connections encode the perception of a familiar odor stimulus. At the onset of the simulation, such synaptic strength existed for a set of PFC neurons that represent familiar odor stimuli. This set includes the neuron $\mathrm{PFC}_{\text {familiar, }}$, which continues firing at high frequency in trial 1 , because the initial spiking caused by input of the familiar odor stimulus (arrow) spreads across recurrent excitatory synaptic connections, causing repeated depolarization and spiking in the same population.

\section{Trial 2}

In trial 2, the perception of a novel odor stimulus elicits an initial spike (arrow) in neurons that are part of the populations containing $\mathrm{EC}_{\text {novel }}$ and $\mathrm{PFC}_{\text {novel }}$. As shown for the neuron $\mathrm{EC}_{\text {novel }}$, spikes elicited by the perception of a novel odor stimulus persists because of the same cholinergic intrinsic reactivation mechanism that maintained activity in trial 1 . In contrast, only a single spike is generated in $\mathrm{PFC}_{\text {novel }}$, because no previously strengthened recurrent connections exist for this neuron in the model. Thus, working memory for the novel stimulus depends on EC in the model.

Trial 3

A lesion of the cholinergic innervation of EC was simulated between trial 2 and trial 3 by removing the ADP currents in simulated EC neurons. The absence of cholinergic modulation prevents sustained spiking in the simulated entorhinal cortex during all subsequent trials. At the start of trial 3, a familiar odor causes an initial spike (arrow) in populations containing $\mathrm{EC}_{\text {familiar }}$ and $\mathrm{PFC}_{\text {familiar }}$, but the absence of cholinergic afterdepolarization prevents persistent spiking in EC. However, the spread of activity across recurrent connections in the PFC allows persistent spiking in $\mathrm{PFC}_{\text {familiar }}$ to maintain a representation of the familiar odor stimulus. This prevents an impairment of matching for the familiar odor stimulus.

\section{Trial 4}

In trial 4, the novel odor causes initial spikes (arrow) in populations containing $\mathrm{EC}_{\text {novel }}$ and $\mathrm{PFC}_{\text {novel. }}$. No self-sustained spiking is possible in the EC because of the simulated lesion of cholinergic innervation removing the afterdepolarization. Because the PFC does not have excitatory recurrent connections representing the novel odor, activity is not maintained in the PFC either. Therefore, this novel odor is forgotten. Our simulation thereby replicates the selective sensitivity of working memory for novel stimuli to the AChX-EC lesion, in contrast to the working memory for familiar stimuli (trial 3).

\section{Trials 5 and 6}

The novel odor stimulus is presented (arrow) at the onset of each following trial. Increasing activity is apparent in the response of neuron $\mathrm{PFC}_{\text {novel }}$, because each presentation causes strengthening by spike timing-dependent plasticity of recurrent excitatory synapses between the neurons in PFC that spike when the novel odor is perceived.

\section{Trials 7 and 8}

Over the previous trials, sufficient synaptic strengthening was achieved for this specific novel stimulus. In trials 7 and 8, persistent spiking is elicited by the perception of the novel odor stimulus, because synapses were strengthened sufficiently during presentations of the novel stimulus odor (in trials 4-6) to enable synaptic reactivation of the set of neurons that include $\mathrm{PFC}_{\text {novel }}$. This demonstrates a mechanism through which a novel odor 
stimulus becomes a familiar odor stimulus after repeated exposure without the support of a buffer in the EC. Our model therefore predicts that gradual synaptic modification resulting from repeated presentation encodes a novel odor stimulus, which offers a mechanistic explanation for the reduction of impairment for novel stimuli that is seen in experiments conducted over multiple days (Fig. 2B).

Our EC buffer model depends on a proposed persistent firing mechanism impaired by a cholinergic lesion. The EC activity could also be disrupted by injections of muscimol into the entorhinal cortex. We predict that encoding of novel odors would be impaired during such injections, causing impairments of matching that would persist even if the muscimol effect wears off during the delay period. Even in the absence of a working EC buffer (resulting from lesions or muscimol injections), our computational model predicts that gradual encoding takes place in intact PFC. We suggest that the gradual encoding mechanism can explain how the performance impairment for novel stimuli is reduced over time. When restricted to a two-item buffer capacity, our model predicts that a reduction of buffer content is caused by the presentation of the same stimuli that elicited maintenance of the last (i.e., second) item in the buffer. Assuming that the buffer contained items A and B and that B was again presented, then the replacement mechanism removes $A$ from the full buffer to make room for new input. Because the input is the same as the remaining buffer content, only one item (B) is sustained by the persistent firing mechanism. Preliminary data from rats with cholinergic lesions of prefrontal cortex show that performance is compromised in the normal DNMS if the subject initially samples the match on the choice phase. In this case, the EC buffer "drops" the sample to store the choice. In the absence of a representation of the sample, response latency increases and accuracy drops to chance. Note that an equivalent prediction applies to a three-item buffer. In addition, the intrinsic mechanism for persistent spiking should be more sensitive to overlap between stimuli than the mechanism using modified synapses. This is consistent with the fact that EC lesions did not change performance with longer lists of odors but predicts that rats with PFC lesions should show greater effects of interference and item drop-out as the difficulty of the task is raised to require simultaneous buffering of more perceived stimuli.

\section{Discussion}

The data presented here demonstrates that selective lesions of the cholinergic innervation of the entorhinal cortex impair delayed nonmatch-to-sample function for novel odors but does not impair the same function for familiar odors. These data indicate a role for cholinergic innervation of the entorhinal cortex in working memory for novel stimuli. Our findings support the hypothesis that intrinsic currents activated by acetylcholine in neurons in entorhinal cortex (Klink and Alonso, 1997b; Egorov et al., 2002) may underlie neuronal activity in this region important for working memory function in delayed matching tasks (Suzuki et al., 1997; Young et al., 1997). The modeling presented here extends previous modeling work (Lisman and Idiart, 1995; Jensen and Lisman, 1996; Fransen et al., 2002; Koene et al., 2003) to demonstrate how loss of cholinergic modulation in the entorhinal cortex could selectively impair delayed nonmatch-to-sample function for novel but not familiar odor stimuli.

Although the AChX-EC lesion rats sustained damage to both the EC and PRh cortex, the impairment in encoding novel stimuli was highly correlated with damage to the EC and not the PRh cortex. The effects of the entorhinal lesion are limited to the encoding of novel stimuli. The AChX-EC rats do not have a general mnemonic problem, as shown by the preservation of memory for familiar items even when mnemonic demands are increased by imposition of a delay (up to $3 \mathrm{~h}$ ) between the sample and choice phase of the task or when the number of items to be remembered was increased (Fig. 3). In addition, the impairment of the AChX-EC rats was not attributable to an increase in task difficulty. The performance of nonlesioned rats showed equivalent changes during memory for new stimuli and during the increase in mnemonic demands when a 10-item list of familiar stimuli was to be remembered. In contrast, AchX-EC rats were impaired relative to SHAMs in memory for new stimuli (Fig. 2) but were not impaired related to SHAMs with the 10-item list of familiar stimuli (Fig. 3). Furthermore, performance on tests with familiar items does not represent recovery of function, because the novel stimuli were tested subsequent to numerous tests with familiar stimuli so that if compensatory mechanisms were in place, the performance for novel items should benefit more than for familiar items.

\section{Relationship to electrophysiological mechanisms}

The selective lesion of cholinergic innervation of the entorhinal cortex may remove mechanisms for sustained spiking activity in entorhinal neurons. Without cholinergic modulation, single entorhinal cortical neurons in slice preparations respond to depolarization with transient spiking activity, which terminates when the input terminates. In contrast, the presence of acetylcholine activates a calcium-sensitive nonspecific cation current, which allows neurons to continue spiking for many minutes after the initial input stimulation has been removed (Klink and Alonso, 1997b; Egorov et al., 2002). Modeling (Fransén et al., 2002) shows how this current could underlie spiking activity during the delay period in delayed matching tasks (Suzuki et al., 1997; Young et al., 1997) as well as phenomena including match enhancement and suppression. The dependence of this current on cholinergic modulation could explain the impairment of matching function with muscarinic cholinergic antagonists (Bartus and Johnson, 1976; Penetar and McDonough, 1983) and the effects shown here.

Although our modeling focuses on sustained spiking activity, the cholinergic enhancement of matching function in this task does not necessarily have to involve sustained spiking during the entire delay. It is unlikely that sustained spiking activity can maintain memory for delays of 15-180 min. The sustained spiking may instead provide an adequate number of spikes to cause sufficient synaptic modification in the entorhinal cortex or hippocampus to allow effective retrieval of an episodic representation of the sample stimulus at the end of the delay period. In this manner, the sustained spiking would provide the same function as the episodic buffer proposed recently on the basis of human experiments (Baddeley, 2000). The selective sensitivity of performance for novel stimuli resembles previous studies that show that NMDA receptor antagonists cause impairments of spatial memory performance in unfamiliar environments, but not in familiar environments (Shapiro and O'Connor, 1992; Caramanos and Shapiro, 1994; Bannerman et al., 1995), and impair encoding of novel items for recognition (Matsuoka and Aigner, 1996).

The hypothesized role of sustained spiking for enhancing encoding of stimuli is consistent with behavioral data showing that blockade of muscarinic cholinergic receptors during encoding impairs performance in a later recognition test (Aigner and Mishkin, 1986; Aigner et al., 1991; Sherman et al., 2003). This impairment also occurs with local infusion of scopolamine (Tang 
et al., 1997) and cholinergic lesions (Turchi et al., 2005) in perirhinal cortex, which is adjacent to entorhinal cortex. Cholinergic activation of a sustained current in perirhinal cortex has not been tested, so impairments from scopolamine infusion could result from spread to entorhinal cortex or effects within perirhinal cortex alone. Cholinergic modulation of spiking activity during delays has not been tested in monkeys.

Neuronal activity in the entorhinal cortex of rats has been analyzed in spatial tasks. Neurons respond over broader spatial locations in the lateral EC (Hargreaves et al., 2005) and in ventromedial regions of the medial EC (Barnes et al., 1990; Frank et al., 2001; Fyhn et al., 2004), consistent with sustained spiking activity initiated at one location persisting through extensive regions. In contrast, more localized spatial firing properties in dorsolateral regions of medial EC suggest that sustained intrinsic spiking may not occur in those regions (Fyhn et al., 2004).

The data do not specify how performance of AChX-EC rats improves with repeated exposure to stimuli. Cholinergic activation of the entorhinal cortex may enhance the relay of information to the hippocampus and thereby facilitate synaptic modification (Hasselmo et al., 2000; McGaughy and Hasselmo, 2004). Loss of cholinergic afferents may simply retard this process rather than block it. Alternatively, the prefrontal or piriform (olfactory) cortex may gradually form representations of odor stimuli independent of the hippocampal representation as the subject becomes aware of the behavioral significance of the stimuli. This process may require spared cholinergic innervation of other cortical regions, which enhances formation of representations of sensory stimuli (Metherate and Weinberger, 1989; Hasselmo, 1995; Dykes, 1997; Weinberger, 2003). Stimulation of nucleus basalis afferents to primary sensory cortex coincident with repeatedly exposed stimuli has been demonstrated previously to induce changes in receptive fields to the paired stimulus in auditory cortex (Weinberger, 2003) and a similar mechanism may exist for other modalities (Dykes, 1997).

\section{Relationship to cognitive neuroscience}

Functional imaging data support the hypothesis that the entorhinal cortex mediates working memory for novel stimuli in contrast to familiar stimuli. Recent imaging studies in humans demonstrate strong activation of parahippocampal structures including the entorhinal cortex during delayed match-to-sample tasks with novel stimuli (Ranganath and D'Esposito, 2001; Stern et al., 2001; Schon et al., 2004; Schon et al., 2005), whereas only prefrontal cortical regions are activated during delayed matching with familiar stimuli. Prefrontal cortex lesions impair DMS tasks with small numbers of highly familiar stimuli (Eacott et al., 1994) but medial temporal lesions do not, whereas medial temporal lesions cause delayed matching impairments for trial unique (i.e., novel) stimuli (Gaffan, 1974; Zola-Morgan et al., 1989, 1993; Alvarez et al., 1994; Leonard et al., 1995). These impairments are less severe with entorhinal lesions than combinations of entorhinal and perirhinal lesions (Leonard et al., 1995), indicating the importance of direct input to the hippocampus from other regions. In rats, medial prefrontal lesions impair delayed matching function for egocentric responses in a T-maze (Aggleton et al., 1995; Ragozzino and Kesner, 2001) and for recurring choices in an eight-arm maze or operant task (Porter et al., 2000), but some researchers argue for its role in attention or behavioral flexibility rather than working memory (Delatour and Gisquet-Verrier, 2000). Lesions of orbital prefrontal cortex impair delayed nonmatch performance more for small numbers of odors, whereas entorhinal-perirhinal lesions impair performance with larger numbers of odors (Otto and Eichenbaum, 1992), indicating that familiar representations in the prefrontal cortex may be important for working memory in conditions of high interference when a small number of items results in greater confusion of item context.

Recent data demonstrate that activity in the entorhinal cortex and other parahippocampal regions during the delay period of a matching task correlates with subsequent memory (Schon et al., 2004). The sustained activity in parahippocampal regions during the delay was reduced by systemic administration of the cholinergic antagonist scopolamine (Schon et al., 2005). This reduction in delay activity suggests that impairments with AChX-EC lesions result from decreases in sustained spiking activity resulting from an absence of acetylcholine. The AChX-EC lesion would not prevent familiar sample stimuli from causing sustained spiking activity in the prefrontal cortex, but the lesion would prevent sustained activity in the entorhinal cortex that could provide working memory for novel stimuli or a buffer for encoding an episodic memory of the encounter with the novel stimulus for subsequent delayed nonmatch performance. Thus, loss of cholinergic activation of sustained spiking could underlie the impairment of delayed nonmatch performance shown here.

\section{References}

Aggleton JP, Neave N, Nagle S, Sahgal A (1995) A comparison of the effects of medial prefrontal, cingulate cortex, and cingulum bundle lesions on tests of spatial memory: evidence of a double dissociation between frontal and cingulum bundle contributions. J Neurosci 15:7270-7281.

Aigner TG, Mishkin M (1986) The effects of physostigmine and scopolamine on recognition memory in monkeys. Behav Neurosci 45:81-87.

Aigner TG, Walker DL, Mishkin M (1991) Comparison of the effects of scopolamine administered before and after acquisition in a test of visual recognition memory in monkeys. Behav Neural Biol 55:61-67.

Alonso A, Garcia-Austt E (1987) Neuronal sources of theta rhythm in the entorhinal cortex of the rat. II. Phase relations between unit discharges and theta field potentials. Exp Brain Res 67:502-509.

Alvarez P, Zola-Morgan S, Squire LR (1994) The animal model of human amnesia: long-term memory impaired and short-term memory intact. Proc Natl Acad Sci USA 91:5637-5641.

Baddeley A (2000) The episodic buffer: a new component of working memory? Trends Cogn Sci 4:417-423.

Bannerman DM, Good MA, Butcher SP, Ramsay M, Morris RG (1995) Distinct components of spatial learning revealed by prior training and NMDA receptor blockade. Nature 378:182-186.

Barnes CA, McNaughton BL, Mizumori SJ, Leonard BW, Lin LH (1990) Comparison of spatial and temporal characteristics of neuronal activity in sequential stages of hippocampal processing. Prog Brain Res 83:287-300.

Bartus RT, Johnson HR (1976) Short term memory in the rhesus monkey: disruption from the anticholinergic scopolamine. Pharmacol Biochem Behav 5:39-40.

Cannon RC, Hasselmo ME, Koene RA (2003) From biophysics to behavior: Catacomb2 and the design of biologically-plausible models for spatial navigation. Neuroinformatics 1:3-42.

Caramanos Z, Shapiro ML (1994) Spatial memory and N-methyl-Daspartate receptor antagonists APV and MK-801: memory impairments depend on familiarity with the environment, drug dose, and training duration. Behav Neurosci 108:30-43.

Delatour B, Gisquet-Verrier P (2000) Functional role of rat prelimbicinfralimbic cortices in spatial memory: evidence for their involvement in attention and behavioural flexibility. Behav Brain Res 109:113-128.

Dudchenko PA, Wood ER, Eichenbaum H (2000) Neurotoxic hippocampal lesions have no effect on odor span and little effect on odor recognition memory but produce significant impairments on spatial span, recognition, and alternation. J Neurosci 20:2964-2977.

Durstewitz D, Seamans JK, Sejnowski TJ (2000) Neurocomputational models of working memory. Nat Neurosci 3:1184-1191.

Dykes RW (1997) Mechanisms controlling neuronal plasticity in somatosensory cortex. Can J Physiol Pharmacol 75:535-545.

Eacott MJ, Gaffan D, Murray EA (1994) Preserved recognition memory for 
small sets, and impaired stimulus identification for large sets, following rhinal cortex ablations in monkeys. Eur J Neurosci 6:1466-1478.

Egorov AV, Hamam BN, Fransen E, Hasselmo ME, Alonso AA (2002) Graded persistent activity in entorhinal cortex neurons. Nature 420:173-178.

Frank LM, Brown EN, Wilson MA (2001) A comparison of the firing properties of putative excitatory and inhibitory neurons from CA1 and the entorhinal cortex. J Neurophysiol 86:2029-2040.

Fransén E, Alonso AA, Hasselmo ME (2002) Simulations of the role of the muscarinic-activated calcium-sensitive nonspecific cation current INCM in entorhinal neuronal activity during delayed matching tasks. J Neurosci 22:1081-1097.

Fyhn M, Molden S, Witter MP, Moser EI, Moser MB (2004) Spatial representation in the entorhinal cortex. Science 305:1258-1264.

Gaffan D (1974) Recognition impaired and association intact in the memory of monkeys after transection of the fornix. J Comp Physiol Psychol 86:1100-1109.

Hargreaves EL, Rao G, Lee I, Knierim JJ (2005) Major dissociation between medial and lateral entorhinal input to dorsal hippocampus. Science 308:1792-1794.

Hasselmo ME (1995) Neuromodulation and cortical function: modeling the physiological basis of behavior. Behav Brain Res 67:1-27.

Hasselmo ME, Fransen E, Dickson C, Alonso AA (2000) Computational modeling of entorhinal cortex. Ann NY Acad Sci 911:418-446.

Hasselmo M, Cannon RC, Koene RA (2002a) A simulation of parahippocampal and hippocampal structures guiding spatial navigation of a virtual rat in a virtual environment: a functional framework for theta theory. In: The parahippocampal region: organisation and role in cognitive functions (Witter MP, Wouterlood FG, eds), pp 139-161. Oxford: Oxford UP.

Hasselmo ME, Bodelon C, Wyble BP (2002b) A proposed function for hippocampal theta rhythm: separate phases of encoding and retrieval enhance reversal of prior learning. Neural Comput 14:793-817.

Jensen O, Lisman JE (1996) Novel lists of 7+/-2 known items can be reliably stored in an oscillatory short-term memory network: interaction with long-term memory. Learn Mem 3:257-263.

Klink R, Alonso A (1997a) Muscarinic modulation of the oscillatory and repetitive firing properties of entorhinal cortex layer II neurons. J Neurophysiol 77:1813-1828.

Klink R, Alonso A (1997b) Ionic mechanisms of muscarinic depolarization in entorhinal cortex layer II neurons. J Neurophysiol 77:1829-1843.

Koene RA, Gorchetchnikov A, Cannon RC, Hasselmo M (2003) Modeling goal-directed spatial navigation in the rat based on physiological data from the hippocampal formation. Neural Netw 16:577-584.

Leonard BW, Amaral DG, Squire LR, Zola-Morgan S (1995) Transient memory impairment in monkeys with bilateral lesions of the entorhinal cortex. J Neurosci 15:5637-5659.

Levy WB, Steward O (1983) Temporal contiguity requirements for longterm associative potentiation/depression in the hippocampus. Neuroscience 8:791-797.

Lisman JE, Idiart MA (1995) Storage of $7+/-2$ short-term memories in oscillatory subcycles. Science 267:1512-1515.

Lisman JE, Fellous JM, Wang XJ (1998) A role for NMDA-receptor channels in working memory. Nat Neurosci 1:273-275.

Markram H, Lubke J, Frotscher M, Sakmann B (1997) Regulation of synaptic efficacy by coincidence of postsynaptic APs and EPSPs. Science 275:213-215.

Matsuoka N, Aigner TG (1996) Cholinergic-glutamatergic interactions in visual recognition memory of rhesus monkeys. NeuroReport 7:565-568.

McGaughy J, Hasselmo ME (2004) High acetylcholine sets circuit dynamics for attention and encoding and low acetylcholine sets dynamics for consolidation. Prog Brain Res 145:207-231.

McGaughy J, Kaiser T, Sarter M (1996) Behavioral vigilance following infusions of 192 IgG-saporin into the basal forebrain: selectivity of the behav- ioral impairment and relation to cortical AChE-positive fiber density. Behav Neurosci 110:247-265.

Metherate R, Weinberger NM (1989) Acetylcholine produces stimulusspecific receptive field alterations in cat auditory cortex. Brain Res 480:372-377.

Otto T, Eichenbaum H (1992) Complementary roles of the orbital prefrontal cortex and the perirhinal-entorhinal cortices in an odor-guided delayed-nonmatching-to-sample task. Behav Neurosci 106:762-775.

Penetar DM, McDonough JH (1983) Effects of cholinergic drugs on delayed match to sample performance of Rhesus monkeys. Pharmacol Biochem Behav 19:963-967.

Porter MC, Burk JA, Mair RG (2000) A comparison of hippocampal or prefrontal cortical lesions on three versions of delayed-non-matching-tosample based on positional or spatial cues. Behav Brain Res 109:69-81.

Ragozzino ME, Kesner RP (2001) The role of rat dorsomedial prefrontal cortex in working memory for egocentric responses. Neurosci Lett 308:145-148.

Ranganath C, D’Esposito M (2001) Medial temporal lobe activity associated with active maintenance of novel information. Neuron 31:865-873.

Schon K, Hasselmo ME, Lopresti ML, Tricarico MD, Stern CE (2004) Persistence of parahippocampal representation in the absence of stimulus input enhances long-term encoding: a functional magnetic resonance imaging study of subsequent memory after a delayed match-to-sample task. J Neurosci 24:11088-11097.

Schon K, Atri A, Hasselmo ME, Tricarico MD, Lopresti ML, Stern CE (2005) Scopolamine reduces persistent activity related to long-term encoding in the parahippocampal gyrus during delayed matching in humans. J Neurosci 25:9112-9123.

Shapiro ML, O'Connor C (1992) N-methyl-D-aspartate receptor antagonist MK-801 and spatial memory representation: working memory is impaired in an unfamiliar environment but not in a familiar environment. Behav Neurosci 106:604-612.

Sherman SJ, Atri A, Hasselmo M, Stern CE, Howard MW (2003) Scopolamine impairs human recognition memory: data and modeling. Behav Neurosci 117:526-539.

Stern CE, Sherman SJ, Kirchhoff BA, Hasselmo ME (2001) Medial temporal and prefrontal contributions to working memory tasks with novel and familiar stimuli. Hippocampus 11:337-346.

Suzuki WA, Miller EK, Desimone R (1997) Object and place memory in the macaque entorhinal cortex. NeuroReport 7:2231-2235.

Swanson LW (1999) Brain maps: structure of the rat brain, p 44. New York: Elsevier.

Tago H, Kimura H, Maeda T (1986) Visualization of detailed acetylcholinesterase fiber and neuron staining in rat brain by a sensitive histochemical procedure. J Histochem Cytochem 34:1431-1438.

Tang Y, Mishkin M, Aigner TG (1997) Effects of muscarinic blockade in perirhinal cortex during visual recognition. Proc Natl Acad Sci USA 94:12667-12669.

Turchi J, Saunders RC, Mishkin M (2005) Effects of cholinergic deafferentation of the rhinal cortex on visual recognition memory in monkeys. Proc Natl Acad Sci USA 102:2158-2161.

Weinberger NM (2003) The nucleus basalis and memory codes: auditory cortical plasticity and the induction of specific, associative behavioral memory. Neurobiol Learn Mem 80:268-284.

Young BJ, Otto T, Fox GD, Eichenbaum H (1997) Memory representation within the parahippocampal region. J Neurosci 17:5183-5195.

Zola-Morgan S, Squire LR, Amaral DG, Suzuki WA (1989) Lesions of perirhinal and parahippocampal cortex that spare the amygdala and hippocampal formation produce severe memory impairment. J Neurosci 9:4255-4270.

Zola-Morgan S, Squire LR, Clower RP, Rempel NL (1993) Damage to the perirhinal cortex exacerbates memory impairment following lesions to the hippocampal formation. J Neurosci 13:251-265. 